\title{
A Design of Electronic Laser Harp Based on SCM
}

\author{
Ling Long ${ }^{\mathrm{a}}$, Zhaoren $\mathrm{Lu}^{\mathrm{b}}$ \\ College of Electrical \& Information Engineering, Southwest University for Nationalities, Chengdu, \\ China \\ along_ling_II@163.com, blu_zhaoren@163.com
}

Keywords: STC89C52RC, Laser Transmitter, Laser Receiving Tube, Modulation Tube.

\begin{abstract}
A design of electronic laser harp based on SCM (the abbreviation for "single chip microcomputer") is proposed. It adopts the semiconductor laser transmitter and the receiving tube as a sensor, and uses the laser transmitter emits beams to imitate the 8 strings of harp. The main control chip STC89C52RC collects and processes the signal from receiving tube circuit, then STC89C52RC outputs special signal to produce sound of harp by audio. Compared with the traditional method, this design greatly enhances the anti-interference ability of the system, and it owns a certain degree of innovation and creation in not only the form of performance but also design proposal of system.
\end{abstract}

\section{Introduction}

A piece of music is made up of many different scales, each scale corresponds to different frequency, and the different note has a specific frequency. Traditional electronic harp uses the analog type photosensitive resistor as the laser beam receiving unit sensor, this design is easily affected by external environment to greatly interfere with the normal operation of system.

In the paper, the design of electronic laser harp used the STC89C52RC as main controller, the sensor of system selected the semiconductor laser transmitter and the receiving tube. Although it's similar to the previous designations such as the electronic organ and electronic guitar, but the selection of sensor and design of circuit structure were largely improved, not only enhanced the design anti-interference of system, but also the design embodied larger innovation in the performance and the train of thought.

\section{The hardware design principles of system}

The system used SCM to generate different frequency signals, which became 8 scales of the laser harp, and 8 semiconductor laser transmitter emitted beams to act as strings. According to if the receiving tube received the level signals by generated a laser beam, SCM would send corresponding frequency square-wave signal to sound the mini audio. The hardware of electronic laser harp consisted of 4 components: the minimum system of SCM, the laser transmitter module, the laser receiver module and the audio sound module.

\section{The minimum system circuit of SCM}

The words "single chip microcomputer" often contracts to "SCM". SCM is a major piece of computer components are integrated into the chip micro - computer to Constitute a complete Microcomputer, these computer components consist of the central processing unit-CPU, the random access memory (RAM), the read-only memory (ROM), the electronically programmable read-only memory (EPROM), the parallel and serial data input-output circuits, the timers/counters, the interrupt controller, and so on ${ }^{[1]}$.

The design selected SCM that is the STC89C52RC made in Hongjing Company. STC89C52RC is based on the 8051 core. Because it adopts CMOS (the abbreviation for "Complementary Metal Oxide Semiconductor") production technology, so it is high-performance 8 bits SCM with low power consumption and cost. STC89C52RC consists of 4 8-bits parallel input-output ports: P0, P1, P2, P3 (32 data lines in total), these data lines are used to input or output data. Moreover, SCM includes 1 
serial input-output port, 3 16-bits timer/counter, 6 grades interrupt system, 21 specific registers, and so on ${ }^{[2]}$.

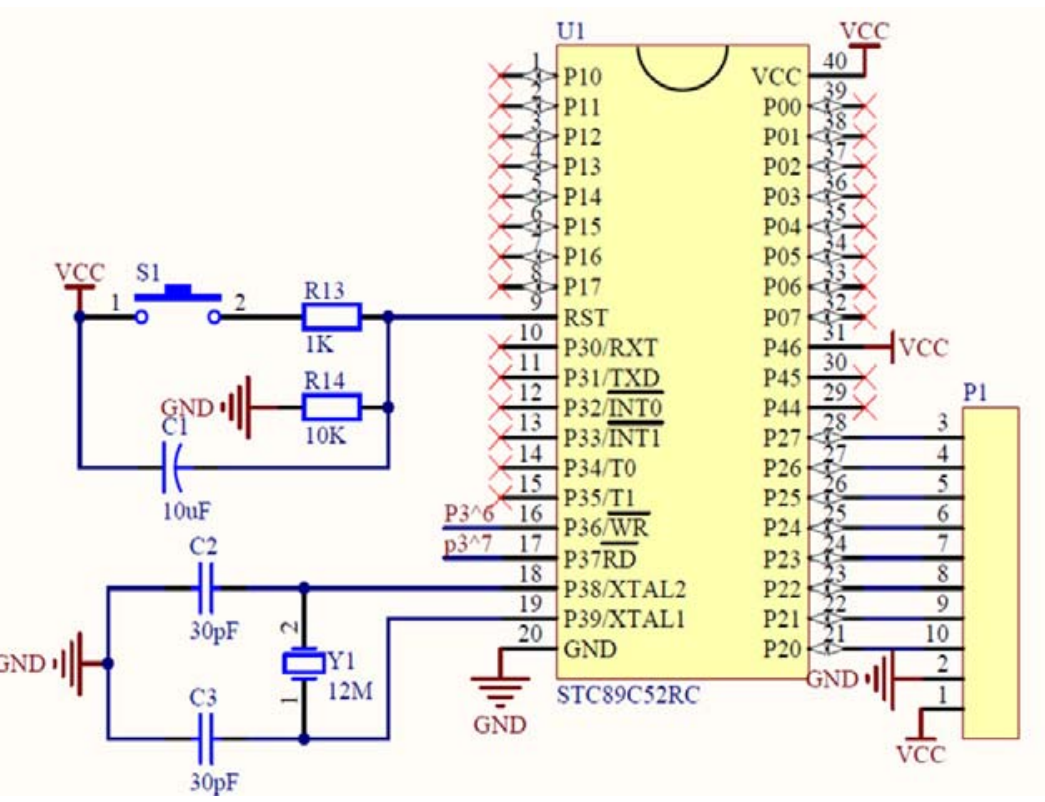

Fig. 1 circuit schematic of STC89C52RC's minimum system

Figure 1 shows that the minimum system circuit was made up of the resistor-condenser reset circuit, the external oscillator circuit and SCM. This design based on SCM's minimum system circuit, the main control circuit made input-output port P2 to connect peripheral device, so system could implement communication between SCM and peripheral laser receiving circuits.

\section{The design of laser transmitting module}

According to the working principle of semiconductor laser transmitter, the semiconductor material shines between the energy bands by driving mode.

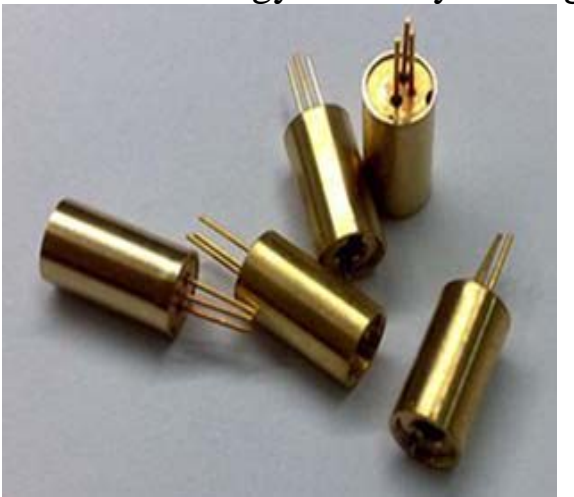

Fig. 2 the physical map of laser transmitter

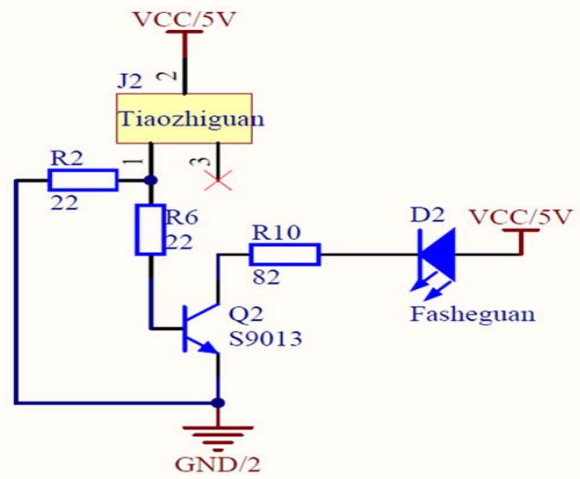

Fig. 3 circuit schematic of laser transmitting module

In the paper, the semiconductor laser transmitter is industrial-grade $25 \mathrm{~mW}$ low-power with external focus. Figure 2 is the physical map of laser transmitter, and figure 3 shows the circuit schematic of laser transmitting module, because design adopted the laser receiving tube that receives only the frequency of $180 \mathrm{KHz}$ laser signals, so the modulation tube $\mathrm{J} 2$ would emitted the $180 \mathrm{KHz}$ signals, which was the square wave signal with duty ratio in $20 \% \sim 30 \%$. The signal made the triode conduction or cutoff at $180 \mathrm{KHz}$, it could modulate frequency emitted by laser transmitter until the laser transmitter emitted laser beams at $180 \mathrm{KHz}^{[3]}$.

\section{The design of laser receiving module}

The function of laser receiving module was to test if laser receiving tube received laser beams with special frequency, and sent corresponding level signal (high or low). System used the level as control signal to sound the mini audio with special frequency. 
The ordinary-low-state laser receiving tube was applied in laser receiving circuit. When laser receiving tube received the $180 \mathrm{KHz}$ laser beams, the second pin of laser receiving tube would output the high level; otherwise it would get the low level.

Because the point-to-point way was used in design; that is to say, a laser receiving tube referred to receive laser beams emitted by a laser transmitter, when something blocked laser beams from someone laser transmitter, the corresponding laser receiving tube would get low level because of receiving nothing. Beyond that, laser receiving tube would output high level. Finally, we got a control signal, i.e., a low level signal we needed.

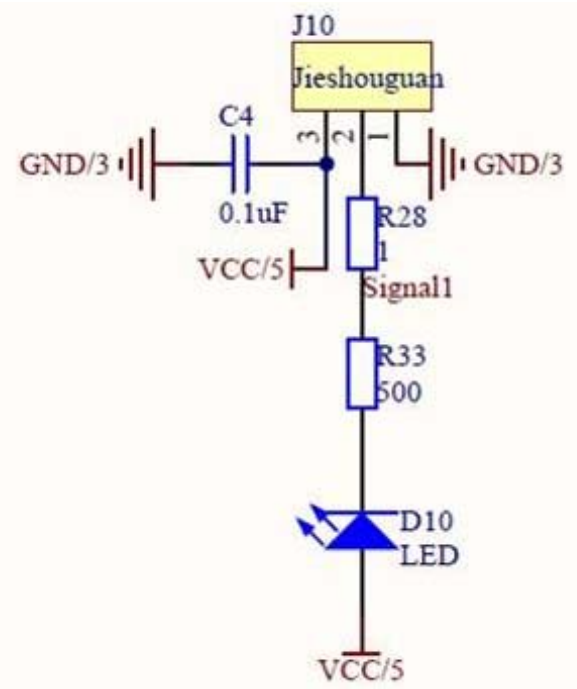

Fig. 4 circuit schematic of laser receiving module

Figure 4 shows the circuit schematic of laser receiving module, the light emitter diode (LED) D10 could get indication if the corresponding laser receiving circuit received laser beams. When the corresponding laser receiving circuit received laser beams, D10 would go out; and when the corresponding laser receiving circuit didn't received laser beams, that's to say, something blocked laser beams, D10 would go on.

\section{The design of sound module}

Instead of using a special driver to sound a speaker, we selected directly an external mini-audio with $3.5 \mathrm{~mm}$ standard audio interface. To do that the hardware circuits of system was largely simplied, and then improved greatly audio quality.
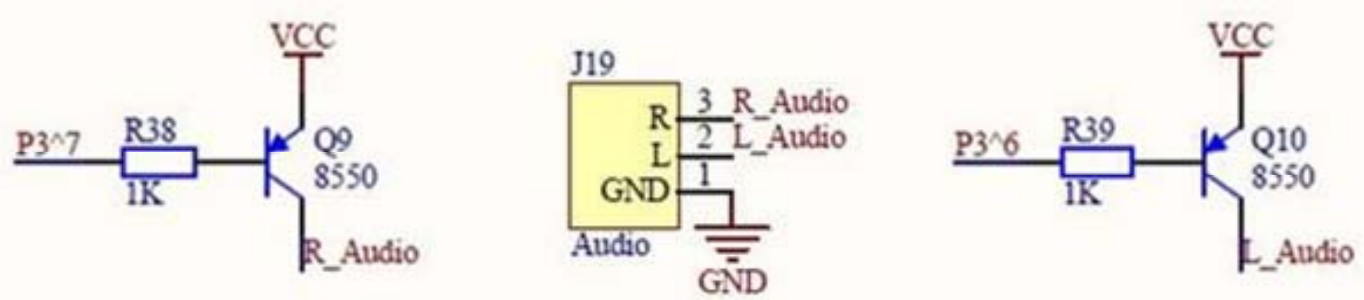

Fig. 5 circuit schematic of audio driver and hardware interface

Figure 5 shows circuit schematic of audio driver and hardware interface, J19 describes a $3.5 \mathrm{~mm}$ standard audio interface. Follow program instructions, when I/O port P3.6 and P3.7 sent a square wave with particular frequency, the triode Q9 and Q10 would be conduction or cutoff follow the particular frequency ${ }^{[4]}$. By doing this, the square wave was sent to mini- audio, and then it would drive mini-audio to sound with an external 5V power "VCC". 


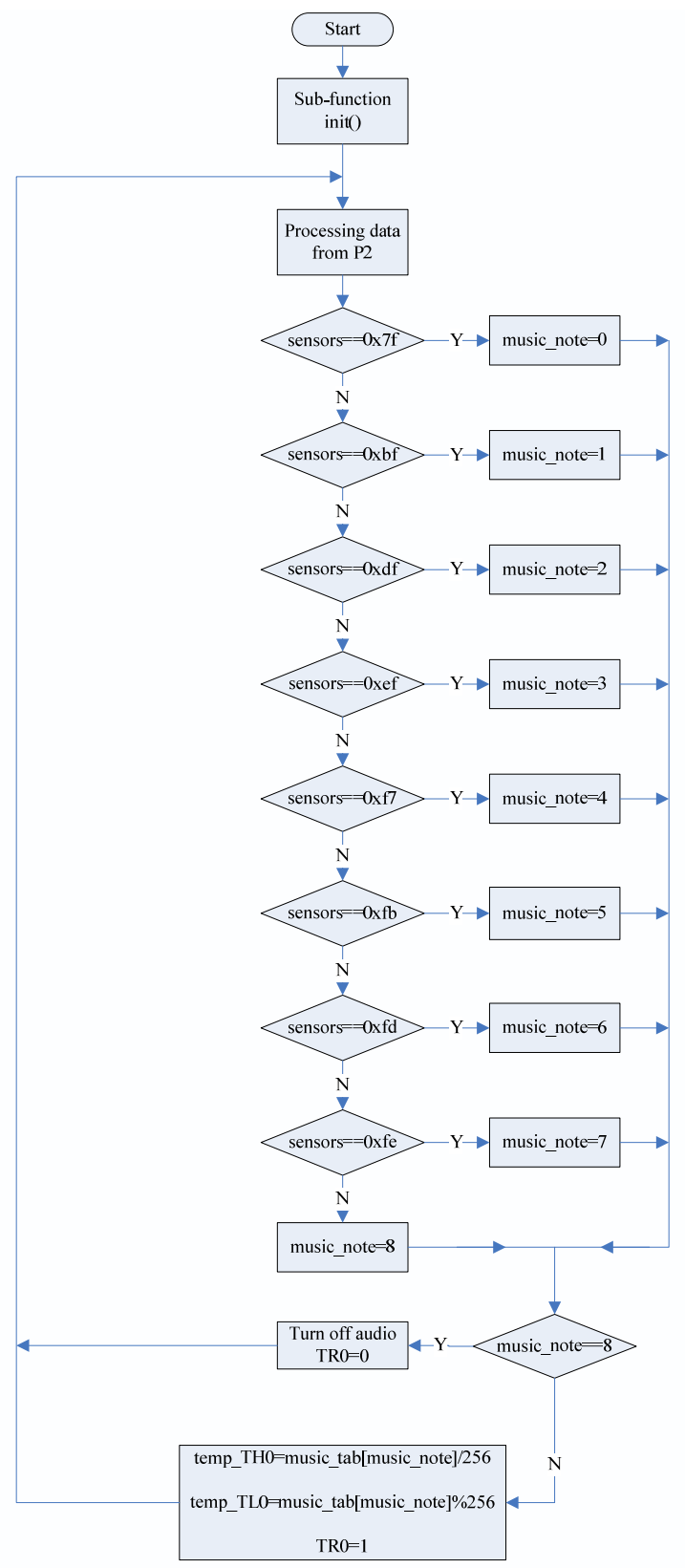

Fig. 6 Main-program flow diagram of system

\section{The program design of system}

In program design, programmer used 51-SCM's own 16-bit timer, which would generate different-frequency square wave with interrupts ${ }^{[5]}$. The whole program includes 1 main function, 1 initialization sub-function and 1 timer interrupting function. Main-program flow diagram is showed in figure 6.

Figure 6 shows that programmer adopted IF statements to test which one of SCM's P2 port output low level. The functions of initialization sub-function were to configure working-mode register (TMOD=0X01) of the timer/counter, to switch on master interrupts $(E A=1)$, and also timer 0 interrupt $(\mathrm{ET} 0=1)$.

In the interrupt function of timer0, P3.6 and P3.7 realized to output square ware with different frequency. Interrupt function of timer0 is showed as below ${ }^{[6]}$.

Void T0_interrupt ( ) interrupt 1

\{

TH0 = temp_TH0; // initial assignment to register.

TLO = temp_TL0; // initial assignment to register. 
amplifier_L $=\sim$ amplifier_L; // to reverse value.

amplifier_R $=\sim$ amplifier_R; // to reverse value.

\}

Interrupt function of timer0 shows that “temp_TH0" and "temp_TL0" are intermediate variables to accomplish initial assignment. Different-frequency square wave would produce by reversing the values, which justly depend on value of intermediate variables “temp_TH0" and "temp_TL0".

Table 1 shows every note and its corresponding frequency and simple-score code.

Tab. 1 comparison table of musical scale

\begin{tabular}{|c|c|c|}
\hline Notes & $\begin{array}{c}\text { Frequency/H } \\
\text { z } \\
\end{array}$ & Simple-core codes \\
\hline Middle 1 do & 523 & 64580 \\
\hline Middle 2 re & 587 & 64684 \\
\hline Middle $3 \mathrm{mi}$ & 659 & 64777 \\
\hline Middle $4 \mathrm{fa}$ & 698 & 64820 \\
\hline Middle 5 so & 784 & 64898 \\
\hline Middle 6 la & 880 & 64968 \\
\hline Middle 7 si & 988 & 65030 \\
\hline High 1 do & 1046 & 65058 \\
\hline
\end{tabular}

\section{Conclusions}

The experiment confirmed: Because of adopting digital laser receiving tube to instead of photosensitive resistance, the design moths greatly weakens external light disturbance. By the point-to-point mode, semiconductor laser transmitter and receiving tube not only largely improve the sound quality of harp, but also strengthen system anti-interference ability. In addition, in the form of performance and design proposal of system, the design solution shows innovative, and the conclusion of the paper has real significance and bright future in the related industry.

\section{Acknowledgements}

This work was financially supported by "the Fundamental Research Funds for the Central Universities” of SWUN (Southwest University for Nationalities) (NO. 12NZYQN10).

\section{References}

[1] Yikun Zhang, Shanjiu Chen, Xuehong Qiu. Principe and Application of Single-Chip Microcomputer, Xian Electronic Science \& Technology University Press, Xian, China, 1998.

[2] Tianxiang Guo. The C Language Tour Based on New Concept 51 Single-Chip Microcomputer for All Strategy: Rudiments, Improvement, Development and Expanding, Electronic Industry Press, Beijing, China, 2009.

[3] Wenlong Zhou, Guowei Xu. Laser Sensor Applies in Intelligent Vehicle Design, Youth Science. 2009 (6): 179-181.

[4] Suxing Yang. Easy - Basic Course of Analog Electronic Technology, third ed., Higher Education Press, Beijing, China, 2006.

[5]Haoqiang Tan. The C Language Program Design, third ed., Qinghua University Press, Beijing, China, 2005.

[6] Prata, S, Yundian Studio (translate), the Chinese C Primer Plus, fifth ed., Posts \& Telecom Press, Beijing, China, 2005. 